\title{
Topological susceptibility in 2-flavor lattice QCD with fixed topololgy
}

\section{T.W. Chiu ${ }^{* a}$, S. Aoki ${ }^{b, c}$, H. Fukaya ${ }^{d}$, S. Hashimoto ${ }^{e, f}$, T.H. Hsieh ${ }^{g}$, T. Kaneko ${ }^{e, f}$, H. Matsufuru ${ }^{e}$, J. Noaki ${ }^{e}$, K. Ogawa ${ }^{a}$, T. Onogi ${ }^{h}$, N. Yamada ${ }^{e, f}$ (JLQCD and TWQCD Collaborations)}

${ }^{a}$ Physics Department, Center for Theoretical Sciences, and National Center for Theoretical Sciences, National Taiwan University, Taipei 10617, Taiwan

${ }^{b}$ Graduate School of Pure and Applied Sciences, University of Tsukuba, Tsukuba 305-8571, Japan

${ }^{c}$ Riken BNL Research Center, Brookhaven National Laboratory, Upton, NY11973, USA

${ }^{d}$ Theoretical Physics Laboratory, RIKEN, Wako 351-0198, Japan

${ }^{e}$ High Energy Accelerator Research Organization (KEK), Tsukuba 305-0801, Japan

${ }^{f}$ School of High Energy Accelerator Science, The Graduate University for Advanced Studies (Sokendai), Tsukuba 305-0801, Japan

${ }^{g}$ Research Center for Applied Sciences, Academia Sinica, Taipei 115, Taiwan

${ }^{h}$ Yukawa Institute for Theoretical Physics, Kyoto University, Kyoto 606-8502, Japan

We determine the topological susceptibility $\chi_{t}$ in the trivial topological sector generated by lattice simulations of two-flavor QCD with overlap Dirac fermion, on a $16^{3} \times 32$ lattice with lattice spacing $\sim 0.12 \mathrm{fm}$, at six sea quark masses $m_{q}$ ranging from $m_{s} / 6$ to $m_{s}$ (where $m_{s}$ is the physical strange quark mass). The $\chi_{t}$ is extracted from the plateau (at large time separation) of the timecorrelation function of the flavor-singlet pseudoscalar meson $\left(\eta^{\prime}\right)$, which arises from the finite size effect due to fixed topology. In the small $m_{q}$ regime, our result of $\chi_{t}$ is proportional to $m_{q}$ as expected from chiral effective theory. Using the formula $\chi_{t}=m_{q} \Sigma / N_{f}$ by Leutwyler-Smilga, we obtain the chiral condensate in $N_{f}=2 \mathrm{QCD}$ as $\Sigma^{\overline{\mathrm{MS}}}(2 \mathrm{GeV})=[254(5)(10) \mathrm{MeV}]^{3}$, in good agreement with our previous result obtained in the $\varepsilon$-regime.

The XXV International Symposium on Lattice Field Theory

July 30 - August 42007

Regensburg, Germany

\footnotetext{
${ }^{*}$ Speaker.

†Email: twchiu@phys.ntu.edu.tw
} 


\section{Introduction}

In Quantum Chromodynamics (QCD), the topological susceptibility $\left(\chi_{t}\right)$ is the most crucial quantity to measure the topological charge fluctuations of the QCD vacuum, which plays an important role in breaking the $U_{A}(1)$ symmetry. Theoretically, $\chi_{t}$ is defined as

$$
\chi_{t}=\int d^{4} x\langle\rho(x) \rho(0)\rangle
$$

where

$$
\rho(x)=\frac{1}{32 \pi^{2}} \varepsilon_{\mu \nu \lambda \sigma} \operatorname{tr}\left[F_{\mu v}(x) F_{\lambda \sigma}(x)\right]
$$

is the topological charge density ( $\propto$ axial anomaly) expressed in term of the matrix-valued field tensor $F_{\mu v}$.

With mild assumptions, Witten [1] and Veneziano [2] obtained a relationship between the topological susceptibility in the quenched approximation and the mass of $\eta^{\prime}$ meson (flavor singlet) in full QCD with $N_{f}$ degenerate flavors, namely, $\chi_{t}$ (quenched) $=f_{\pi}^{2} m_{\eta^{\prime}}^{2} /\left(4 N_{f}\right)$ where $f_{\pi}=131$ $\mathrm{MeV}$, the decay constant of pion. This implies that the mass of $\eta^{\prime}$ is essentially due to the axial anomaly relating to non-trivial topological charge fluctuations, which can turn out to be nonzero even in the chiral limit, unlike those of the (non-singlet) approximate Goldstone bosons.

Using the Chiral Perturbation Theory (ChPT), Leutwyler and Smilga [3] obtained the following relation in the chiral limit,

$$
\chi_{t}=\frac{m_{q} \Sigma}{N_{f}}+\mathscr{O}\left(m_{q}^{2}\right)
$$

where $m_{q}$ is the quark mass, and $\Sigma$ is the chiral condensate. In other words, as $m_{q} \rightarrow 0$, the topological susceptibility is suppressed due to internal quark loops. Most importantly, (1.2) provides a viable way to extract $\Sigma$ from $\chi_{t}$ in the chiral limit.

From (1.1), one obtains

$$
\chi_{t}=\frac{\left\langle Q_{t}^{2}\right\rangle}{\Omega}, \quad Q_{t} \equiv \int d^{4} x \rho(x)
$$

where $\Omega$ is the volume of the system, and $Q_{t}$ is the topological charge (which is an integer for QCD). Thus, one can obtain $\chi_{t}$ by counting the number of gauge configurations for each topological sector. Obviously, for a set of gauge configurations in the topologically-trivial sector with $Q_{t}=0$, it gives $\chi_{t}=0$. However, even for a topologically-trivial gauge configuration, it may possess non-trivial topological excitations in sub-volumes. Thus, one can investigate whether there are topological excitations within any sub-volumes, and to measure $\chi_{t}$ using the correlation of the topological charges of two sub-volumes [4].

In general, for any topological sector with $Q_{t}$, using saddle point expansion on the QCD partition function in a finite volume, it can be shown that [5] (see also [6])

$$
\lim _{|x| \rightarrow \infty}\langle\rho(x) \rho(0)\rangle=\frac{1}{\Omega}\left(\frac{Q_{t}^{2}}{\Omega}-\chi_{t}-\frac{c_{4}}{2 \chi_{t} \Omega}\right)+\mathscr{O}\left(\Omega^{-3}\right)
$$


where

$$
c_{4}=-\frac{1}{\Omega}\left[\left\langle Q_{t}^{4}\right\rangle_{\theta=0}-3\left\langle Q_{t}^{2}\right\rangle_{\theta=0}^{2}\right]
$$

Thus, one can consider two spatial sub-volumes at time slices $t_{1}$ and $t_{2}$, and to measure their time-correlation function

$$
C\left(t_{1}-t_{2}\right)=\left\langle Q\left(t_{1}\right) Q\left(t_{2}\right)\right\rangle=\sum_{\overrightarrow{x_{1}}, \overrightarrow{x_{2}}}\left\langle\rho\left(x_{1}\right) \rho\left(x_{2}\right)\right\rangle
$$

where the summations run over the spatial volumes at $t_{1}$ and $t_{2}$ respectively. Then its plateau at large $\left|t_{1}-t_{2}\right|$ can be used to extract $\chi_{t}$, provided that $\left|c_{4}\right| \ll 2 \chi_{t}^{2} \Omega$.

However, for lattice $\mathrm{QCD}$, it is difficult to extract $\rho(x)$ and $Q_{t}$ unambiguously from the gauge link variables, due to their rather strong fluctuations.

To circumvent this difficulty, one may consider the Atiyah-Singer index theorem [7]

$$
Q_{t}=n_{+}-n_{-}=\operatorname{index}(\mathscr{D})
$$

where $n_{ \pm}$is the number of zero modes of the massless Dirac operator $\mathscr{D} \equiv \gamma_{\mu}\left(\partial_{\mu}+i g A_{\mu}\right)$ with \pm chirality. Since $\mathscr{D}$ is anti-Hermitian and chirally symmetric, its nonzero eigenmodes must come in complex conjugate pairs (i.e., $\mathscr{D} \phi=i \lambda \phi$ implies $\mathscr{D} \gamma_{5} \phi=-i \lambda \gamma_{5} \phi$, for $\lambda=\lambda^{*} \neq 0$ ) with zero chirality $\left(\int d^{4} x \phi^{\dagger} \gamma_{5} \phi=0\right)$. Thus one can obtain the identity

$$
n_{+}-n_{-}=\int d^{4} x m \operatorname{tr}\left[\gamma_{5}(\mathscr{D}+m)^{-1}(x, x)\right]
$$

by spectral decomposition, where the nonzero modes drop out due to zero chirality. In view of (1.4) and (1.5), one can regard $m \operatorname{tr}\left[\gamma_{5}(\mathscr{D}+m)^{-1}(x, x)\right]$ as topological charge density, to replace $\rho(x)$ in the measurement of $\chi_{t}$.

For lattice QCD, it is well-known that the overlap Dirac operator [8] in a topological non-trivial gauge background possesses exact zero modes (with definite chirality) satisfying the Atiyah-Singer index theorem. Writing the massive overlap Dirac operator as

$$
D(m)=\left(m_{0}+\frac{m}{2}\right)+\left(m_{0}-\frac{m}{2}\right) \gamma_{5} \frac{H_{w}\left(-m_{0}\right)}{\sqrt{H_{w}^{2}\left(-m_{0}\right)}}
$$

where $H_{w}\left(-m_{0}\right)$ is the standard Hermitian Wilson operator with negative mass $-m_{0}\left(0<m_{0}<2\right)$, then the topological charge density can be defined as

$$
\rho_{1}(x)=m \operatorname{tr}\left[\gamma_{5}\left(D_{c}+m\right)_{x, x}^{-1}\right]
$$

where $\left(D_{c}+m\right)^{-1}$ is the valence quark propagator with quark mass $m$, and $D_{c}$ is a chirally symmetric and non-local operator, relating to $D(0)$ by $D_{c}=D(0)\left[1-D(0) /\left(2 m_{0}\right)\right]^{-1}$ [9]. Note that $\left(D_{c}+m\right)^{-1}$ is exponentially-local for sufficiently smooth gauge background and nonzero $m$. Here $\rho_{1}(x)$ is justified to be topological charge density, since it can be shown that (see e.g., [10])

$$
\sum_{x} \rho_{1}(x)=m \operatorname{Tr}\left[\gamma_{5}\left(D_{c}+m\right)_{x, x}^{-1}\right]=n_{+}-n_{-}
$$


which is similar to its counterpart in continuum (1.5), where $\operatorname{Tr}$ denotes trace over Dirac, color and lattice spaces.

Now we can replace $\rho(x)$ with $\rho_{1}(x)$, and use (1.3) to extract $\chi_{t}$ for any topological sector. However, on a finite lattice, it is contaminated by $m_{\pi}, m_{\eta^{\prime}}$ and any states which can couple to $\left\langle\rho_{1}(x) \rho_{1}(0)\right\rangle$. A better alternative is to compute the correlator of the flavor-singlet pseudoscalar meson $\eta^{\prime}$, which behaves as $[4,5]$

$$
\lim _{|x| \gg 1} m_{q}^{2}\left\langle\eta^{\prime}(x) \eta^{\prime}(0)\right\rangle \simeq \frac{1}{\Omega}\left(\frac{Q_{t}^{2}}{\Omega}-\chi_{t}-\frac{c_{4}}{2 \chi_{t} \Omega}\right)+\mathscr{O}\left(e^{-m_{\eta^{\prime}}|x|}\right)+\mathscr{O}\left(\Omega^{-3}\right)
$$

Then the time-correlation function of $\eta^{\prime}$ (see Fig. 1(a)) is fitted to $A+B\left(e^{-M t}+e^{-M(T-t)}\right)$ to extract the constant $A=\frac{1}{m_{q}^{2}} \frac{1}{T}\left(\frac{Q_{t}^{2}}{\Omega}-\chi_{t}-\frac{c_{4}}{2 \chi_{t} \Omega}\right)$ and $\chi_{t}$, provided that $\left|c_{4}\right| \ll 2 \chi_{t}^{2} \Omega$.

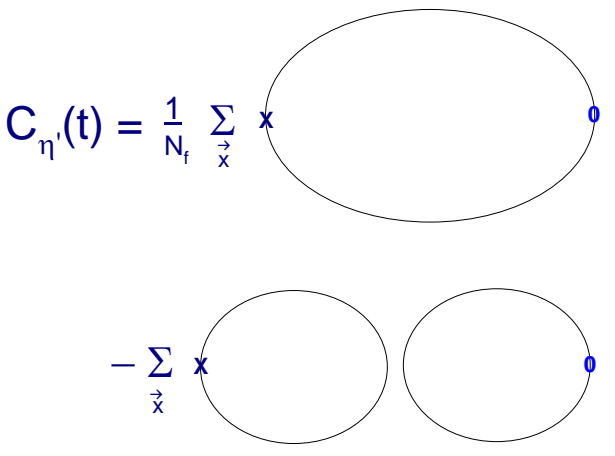

(a)

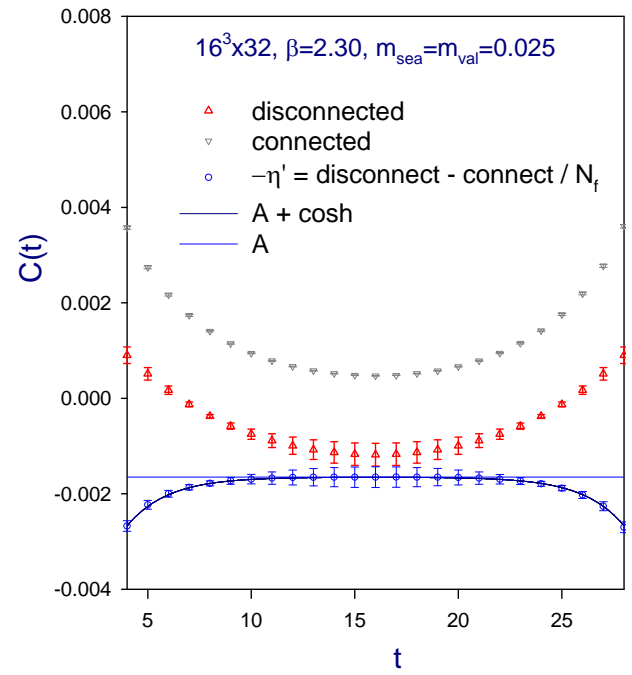

(b)

Figure 1: (a) A schematic diagram for the time-correlation function of the flavor singlet pseudoscalar meson operator. Each solid line denotes the valence quark propagator $\left(D_{c}+m_{q}\right)^{-1}$. (b) The time-correlation function of the flavor singlet (circles), and its connected (triangle down) and disconnected (triangle up) contributions. Data for $m_{q}=0.025$ are shown.

\section{Lattice Setup}

Simulations are carried out for two-flavor $\left(N_{f}=2\right)$ QCD on a $16^{3} \times 32$ lattice at a lattice spacing $\sim 0.12 \mathrm{fm}$. For the gluon part, the Iwasaki action is used at $\beta=2.30$, together with unphysical Wilson fermions and associated twisted-mass ghosts [11]. The unphysical degrees of freedom generate a factor $\operatorname{det}\left[H_{w}^{2}\left(-m_{0}\right) /\left(H_{w}^{2}\left(-m_{0}\right)+\mu^{2}\right)\right]$ in the partition function (we take $m_{0}=1.6$ and $\mu=0.2)$ that suppresses the near-zero eigenvalue of $H_{w}\left(-m_{0}\right)$ and thus makes the numerical operation with the overlap operator substantially faster. Furthermore, since the exact zero eigenvalue is forbidden, the global topological change is preserved during the molecular dynamics evolution of 
the gauge field. Our main runs are performed at $Q=0$, while $Q=-2$ and -4 configurations are also generated at one sea quark mass in order to check the consistency as described below.

For the sea quark mass $m_{q}$ we take six values: $0.015,0.025,0.035,0.050,0.070$, and 0.100 that cover the mass range $m_{s} / 6-m_{s}$ with $m_{s}$ the physical strange quark mass. After discarding 500 trajectories for thermalization, we accumulate 10,000 trajectories in total for each sea quark mass. In the calculation of $\chi_{t}$, we take one configuration every 20 trajectories, thus we have 500 configurations for each $m_{q}$. For each configuration, 50 pairs of lowest-lying eigenmodes of the overlap-Dirac operator $D(0)$ are calculated using the implicitly restarted Lanczos algorithm and stored for the later use.

\section{Results}

For the connected diagram (see Fig. 1(a)), the pion correlator is computed using the conjugate gradient algorithm with a low-mode preconditioning. Low-modes are also used for averaging over source points [12], which significantly improves the statistical signal. For the disconnected diagram, the quark propagator is represented by the eigenmode decomposition and approximated by the 50 conjugate pairs of the low-lying eigenmodes. The quark propagator is then obtained for any source point without extra computational cost, and the disconnected loops can be calculated with an average over the source point. The truncation is motivated by the expectation that the long distance correlation is dominated by the low-lying fermion modes; its validity has to be checked numerically (see below).

In Fig. 1(b), we plot $-C_{\eta^{\prime}}(t)$ together with those of connected and disconnected parts for $m_{q}=0.025$. The curve is a fit to a function $A+B\left(e^{-M t}+e^{-M(T-t)}\right)$ with data for $-C_{\eta^{\prime}}(t)$ in the range $t \in[4,28]$. The horizontal line is a fitted constant $A=1.70(13) \times 10^{-3}$, where the error is estimated using the jackknife method with bin size of 20 configurations, with which the statistical error saturates. Assuming $\left|c_{4}\right| \ll 2 \chi_{t}^{2} \Omega$, we obtain $a^{4} \chi_{t}=3.40(27) \times 10^{-5}$ at $m_{q}=0.025$.

Since the disconnected diagram is computed with only 50 pairs of low-lying eigenmodes, we have to check whether they suffice to saturate $C_{\eta^{\prime}}(t)$. For the time range $[4,28]$ used for fitting, as the number of eigenmodes is increased from 10 to 30 , the change of correlator $\left|\delta C_{\eta^{\prime}}\right| / C_{\eta^{\prime}}$ is $\sim 3 \%$, while from 30 to 50 , it is only $\sim 0.3 \%$, which is less than $8 \%$ of the statistical error. Thus $C_{\eta^{\prime}}$ is well saturated with 50 eigenmodes. This also holds for all six sea quark masses.

In Fig. 2, we plot the topological susceptibility $\chi_{t} a^{4}$ as a function of the sea quark mass $m_{q} a$, The statistical precision is good enough to find a clear dependence on the sea quark mass. For the smallest three quark masses, 0.015, 0.025, and 0.035, the data are well fitted by a linear function $F+G m$ with the intercept $F=0.0(1) \times 10^{-5}$ and the slope $G=0.00133(5)$. Evidently, the intercept is consistent with zero, in agreement with the $\chi \mathrm{PT}$ expectation (1.2). Equating the slope to $a^{3} \Sigma / N_{f}$, we obtain $a^{3} \Sigma=0.0027(1)$. In order to convert $\Sigma$ to that in the $\overline{\mathrm{MS}}$ scheme, we calculate the renormalization factor $Z_{m}^{\overline{\mathrm{MS}}}(2 \mathrm{GeV})$ using the non-perturbative renormalization technique through the RI/MOM scheme [13]. Our result is $Z_{m}^{\overline{\mathrm{MS}}}(2 \mathrm{GeV})=0.742(12)$ [14]. With $a^{-1}=1670(20)(20) \mathrm{MeV}$ determined with $r_{0}=0.49 \mathrm{fm}$ [15], the value of $\Sigma$ is transcribed to $\Sigma^{\overline{\mathrm{MS}}}(2 \mathrm{GeV})=[254(5)(10) \mathrm{MeV}]^{3}$, which is in good agreement with our previous result $\left[251(7)(11) \mathrm{MeV}^{3}[16]\right.$ obtained in the $\varepsilon$-regime from the low-lying eigenvalues. The errors represent a combined statistical error $\left(a^{-1}\right.$ and $\left.Z_{m}^{\overline{\mathrm{MS}}}\right)$ and the systematic error estimated from the higher 


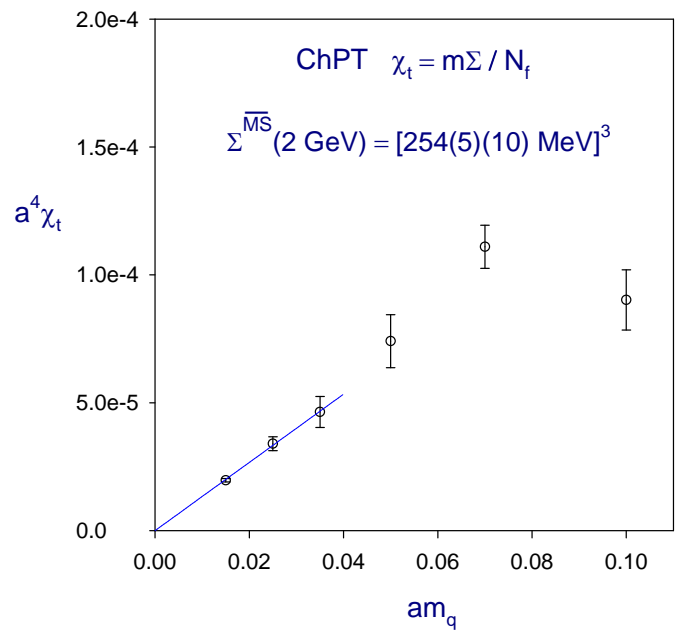

Figure 2: Topological susceptibility $\chi_{t} a^{4}$ versus sea quark mass $m_{q} a$.

order effects (e.g., $c_{4}$ term), respectively. Since the calculation is done at a single lattice spacing, the discretization error cannot be quantified reliably, but we do not expect much larger error because our lattice action is free from $O(a)$ discretization effects. Our results of topological susceptibility are listed in Table 1, for six sea quark masses respectively.

\begin{tabular}{|c|cccccc|}
\hline$m_{q} r_{0}$ & 0.0614 & 0.1023 & 0.1432 & 0.2046 & 0.2861 & 0.4091 \\
\hline$\chi_{t} r_{0}^{4}$ & $0.0055(2)$ & $0.0095(8)$ & $0.0130(17)$ & $0.0212(29)$ & $0.0313(26)$ & $0.0253(33)$ \\
\hline
\end{tabular}

Table 1: The values of topological susceptibility $\chi_{t} r_{0}^{4}$ extracted in the $Q=0$ sector, for six sea quark masses respectively.

In principle, $\chi_{t}$ in (1.3) is universal for any topological sector. We check the universality of $\chi_{t}$ as follows. At sea quark mass $m_{q}=0.050$, we generate 250 configurations with $Q=-2$ and -4 respectively in addition to the main run at $Q=0$. Then we extract $\chi_{t}$ from the time-correlation function of $\eta^{\prime}$, similar to the $Q=0$ case. Our results for $a^{4} \chi_{t}$ are: $\{7.4(1.3), 6.4(2.1), 5.9(1.8)\} \times$ $10^{-5}$ for $Q=\{0,-2,-4\}$ respectively. Evidently, $\chi_{t}$ extracted from different topological sectors are consistent with each other within the statistical error.

Finally, we come to the assumption $\left|c_{4}\right| \ll 2 \chi_{t}^{2} \Omega$ in extracting $\chi_{t}$ via (1.3). With the formulas derived in [5], we can obtain an estimate of the upper bound of $\left|c_{4}\right| /\left(2 \chi_{t}^{2} \Omega\right)$ by measuring the two-point correlator $\left\langle\rho_{1}\left(x_{1}\right) \rho_{1}\left(x_{2}\right)\right\rangle$ and the four-point correlator $\left\langle\rho_{1}\left(x_{1}\right) \rho_{1}\left(x_{2}\right) \rho_{1}\left(x_{3}\right) \rho_{1}\left(x_{4}\right)\right\rangle$. Our preliminary result is $\left|c_{4}\right| /\left(2 \chi_{t}^{2} \Omega\right)<0.1$, for all six sea quark masses. Details of this calculation will be presented elsewhere. We note that the upper bound $\left|c_{4}\right| /\left(2 \chi_{t}^{2} \Omega\right)<0.1$ is also consistent with the ratio $c_{4} / \chi_{t} \simeq 0.3$ obtained in the quenched approximation [17].

\section{Conclusion}

In this paper, we have determined the topological susceptibility $\chi_{t}$ in two-flavor QCD from a lattice calculation of two-point correlators at a fixed global topological charge $Q_{t}=0$. The expected 
sea quark mass dependence of $\chi_{t}$ from $\chi \mathrm{PT}$ is clearly observed with the good statistical precision we achieved, in contrast to the previous unquenched lattice calculations. Our result indicates that the topologically non-trivial excitations (e.g., instanton and anti-instanton pairs) are in fact locally active in the QCD vacuum, even when the global topological charge is kept fixed. The information of these topological excitations is carried by low-lying fermion eigenmodes if the exact chiral symmetry is preserved on the lattice. This work demonstrates that Monte Carlo simulation of lattice QCD with fixed topology is a viable approach, to be pursued when the topology change hardly occurs near the continuum limit even with chirally non-symmetric lattice fermions. The artifacts due to the fixed topology in a finite volume can be removed to obtain the physics results in the $\theta$ vacuum, provided that $\chi_{t}$ has been determined in the first place $[5,6]$, as has been done in this work.

Numerical simulations are performed on Hitachi SR11000 and IBM System Blue Gene Solution at High Energy Accelerator Research Organization (KEK) under a support of its Large Scale Simulation Program (No. 07-16), and also in part on NEC SX-8 at YITP (Kyoto U), NEC SX-8 at RCNP (Osaka U), and IBM/HP clusters at NCHC and NTU-CC (Taiwan). This work is supported in part by the Grant-in-Aid of the Japanese Ministry of Education (No. 13135204, 15540251, $17740171,18034011,18340075,18740167,18840045,19540286$, and 19740160) and the National Science Council of Taiwan (No. NSC95-2112-M002-005, NSC95-2112-M001-072).

\section{References}

[1] E. Witten, Nucl. Phys. B 156, 269 (1979).

[2] G. Veneziano, Nucl. Phys. B 159, 213 (1979).

[3] H. Leutwyler and A. Smilga, Phys. Rev. D 46, 5607 (1992).

[4] H. Fukaya and T. Onogi, Phys. Rev. D 70, 054508 (2004)

[5] S. Aoki, H. Fukaya, S. Hashimoto and T. Onogi, Phys. Rev. D 76, 054508 (2007)

[6] R. Brower, S. Chandrasekharan, J. W. Negele and U. J. Wiese, Phys. Lett. B 560, 64 (2003)

[7] M. F. Atiyah and I. M. Singer, Annals Math. 87, 484 (1968).

[8] H. Neuberger, Phys. Lett. B 417, 141 (1998); Phys. Lett. B 427, 353 (1998)

[9] T. W. Chiu and S. V. Zenkin, Phys. Rev. D 59, 074501 (1999)

[10] T. W. Chiu and T. H. Hsieh, Phys. Rev. D 66, 014506 (2002)

[11] H. Fukaya, S. Hashimoto, K. I. Ishikawa, T. Kaneko, H. Matsufuru, T. Onogi and N. Yamada [JLQCD Collaboration], Phys. Rev. D 74, 094505 (2006)

[12] T. A. DeGrand and S. Schaefer, Comput. Phys. Commun. 159, 185 (2004)

[13] G. Martinelli, C. Pittori, C. T. Sachrajda, M. Testa and A. Vladikas, Nucl. Phys. B 445, 81 (1995)

[14] J. Noaki et al. [JLQCD Collaboration], PoS LAT2007, 126 (2007), arXiv:0710.0929 [hep-lat].

[15] T. Kaneko et al. [JLQCD Collaboration], PoS LAT2006, 054 (2006)

[16] H. Fukaya et al. [JLQCD Collaboration], Phys. Rev. Lett. 98, 172001 (2007) H. Fukaya et al. [JLQCD and TWQCD Collaborations] Phys. Rev. D 76, 054503 (2007)

[17] L. Giusti, S. Petrarca and B. Taglienti, arXiv:0705.2352 [hep-th] L. Del Debbio, H. Panagopoulos and E. Vicari, arXiv:0706.1479 [hep-th] 